REVIEW

\title{
Use of the single subject design for practice based primary care research
}

\section{J E Janosky}

Postgrad Med J 2005;81:549-551. doi: 10.1136/pgmj.2004.031005

The use of a single subject research design is proposed for practice based primary care research. An overview of the rationale of the design, an introduction to the methodology, strengths, limitations, a sample of recent literature citations, a working example, and possible clinical applications are presented.

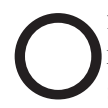
ften overlooked in primary care research is the single subject research design. A single subject research design can be used to study the time course, variability, or effect of an intervention or treatment on a single patient. This experimental research design involving multiple measurements over time on a single subject $(\mathrm{n}=1)$ has been labelled as a clinical trial of $\mathrm{n}=1$, a randomised clinical trial in a single patient, a within subject design, patient care study, A-B, or a single subject design. ${ }^{1-9}$

When conducting patient care, a physician is most probably treating the patient using a single subject paradigm. Typically, a patient presents to a physician with a complaint or symptom. As a result of the history, examination, and medical testing by the physician, for the patient's presenting complaint, an intervention or treatment might be started. Evaluation is then made of the effectiveness of the treatment in eliminating or modifying the complaint or underlying identified condition. When conducting a single subject research design as compared with patient care, sound research methodology would need to be included. This would include standardisation of the intervention including the assessment of compliance, objective measurement of the outcomes, procedures to minimise bias such as blinding or second observers, and so forth. With the implementations, it can be argued that patient care is a special case of a single subject research design.

The randomised parallel group clinical trial design is usually considered as the gold standard in clinical research. The results of these trials provide information on the average treatment effects for the studied populations and serve as guidelines for evidence based medicine. At times, the results from these trials might not always be applicable in determining the most effective treatment for an individual patient: (1) patients might be different clinically from those that participated in the trial, (2) the heterogeneity of the patients in the trial may limit the generalisability of the results to particular patient populations, and (3) the strict criteria for trial participation might be of limited applicability to a general clinic patient populations. To examine the applicability of the results of a clinical trial to a specific patient, a single subject research design could be used.

An Institute of Medicine report ${ }^{10}$ has provided some guidelines as to the use of these small clinical trials. Specifically, warranted situations might include rare diseases, unique study populations, individually tailored treatments, isolated environments, emergency situations, and public health urgency. ${ }^{10}$ Practice based primary care research, commonly, encompasses individually tailored treatments (for example, glycaemic control), isolated environments (for example, rural health), and unique study populations (for example, HIV infected pregnant adolescent).

\section{DESIGN OVERVIEW}

A patient care or an experimental single subject research design can have multiple periods of measurement and multiple times for intervention or treatment. At the onset of each of these designs, a series of baseline (A) observations are taken to assess the patient or subject in the initial or diseased state. A course of treatment is prescribed, denoted by the treatment or (B) phase. The patient or subject continues with the treatment, while assessing the effectiveness across time, using the same outcome variables that were used during the baseline phase. This describes the primary A-B single subject design. Other designs might include removal of the intervention or treatment referred to as washouts, the second baseline (A), a readministration of the treatment (B), or different treatments (C). The research question of interest should guide the single subject design used in terms of combinations of baselines and treatments (A-B, A-B-A, A-B-A-B, A-B-C, etc). Depending upon the research question of interest, varying combinations of phases of observation consisting of baseline, washout, intervention, and so forth. ${ }^{11}$ If more than one treatment is planned, the order of the treatments may be randomised; and, where feasible, the researcher and patient are blinded to the order of the treatments. Within each of these phases, multiple across time observations are obtained. If there are multiple repeating phases, these are considered as periods. One, or more than one, outcome variable(s) may be measured.

\section{A WORKING EXAMPLE}

As an example of the use of a single subject research design in clinical practice, a person with diabetes might present to their family physician for additional disease and treatment 


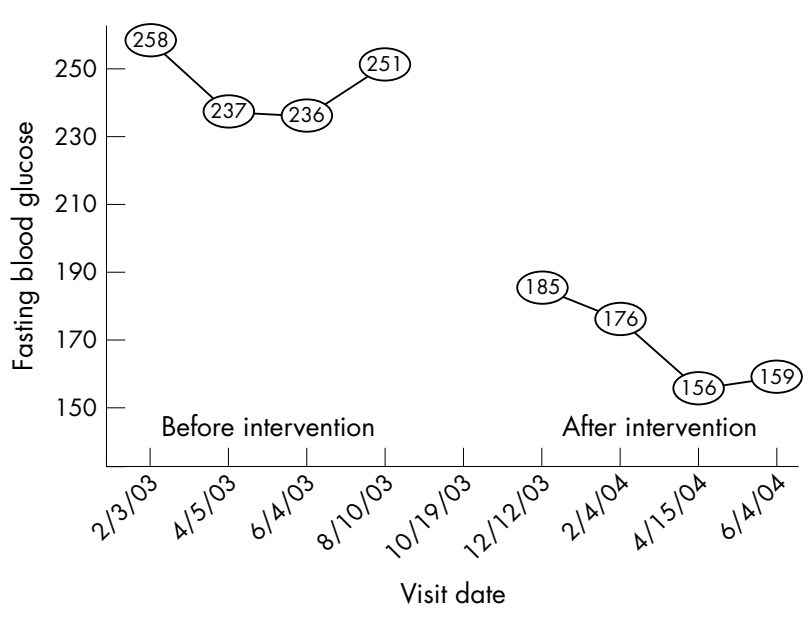

Figure 1 An illustration of an A-B single subject research design.

management. In the language of a single subject design, the initial reporting of the patient to their family physician, and the physician's evaluation of the patient is considered the baseline phase. This phase, of a single subject design, encompasses an evaluation of the initial state of the patient. For this diabetic patient, this phase would consist of review of the past blood glucose readings and some initial testing (for example, $\mathrm{HbAlC}$ ). For a single subject design, the period of time when the patient is receiving an intervention is referred to as the treatment phase. For this diabetic patient, that could include an insulin adjustment, a knowledge based diabetic education course, a behavioural intervention, and others. The assessment of the effectiveness of the treatment is then made by a comparison of the initial with the treatment phase. ${ }^{12}$ If the first treatment was not effective, a choice might be made to stop the initial treatment or change to a new treatment, or both, thus introducing another baseline or treatment phase into the design. During each of these phases, one or more measurements might be taken to assess the course or variability in the response, which entails multiple measurements of $\mathrm{HbAlC}$ at spaced visits. In a single subject design, the typical number of measurements within a phase is seven. ${ }^{9}$

Presented in figure 1 is an illustration of an implemented A-B single subject research design. The research question of interest for this study was whether a comprehensive intervention for diabetes management would be effective in lowering the fasting blood glucose values. In choosing the patient to participate in this single subject study, careful consideration would be given to identify a patient that would be considered as:

(1) typical in terms of the practice demographics,

(2) typical for the disease presentation and progression,

(3) in need of lower fasting blood glucose values,

(4) anticipated to be compliant for the treatment changes,

(5) anticipated to be compliant for the necessary outcome assessments, and

(6) would give informed consent.

For this particular single subject design, there are two phases to this design: a baseline (A) and a treatment (B). The baseline and treatment are both administered one time. There are measurements across time, within both the baseline and treatment phases, the patient was seen every two months for a fasting blood glucose measurement.

Presented in figure 1 are the data for the fasting blood glucose values. The patient was a 57 year old white woman, with an onset of insulin dependent diabetes in 1992. Presented are four measurements, every two months, before and after the intervention. A total of eight observations are presented, and each observation is measured and reported (for example, laboratory used, time of day, etc) in the same manner. The intervention consisted of a prescribed exercise regimen, weight management, and participation in a counselling session that was goaled to present diabetes management. As can be seen, it appears that the intervention was effective in lowering the measured fasting blood glucose in this subject. As in all single subject designs, the research question of interest should guide the single subject design used.

\section{RECENT EXAMPLES FROM LITERATURE}

Fisher, although most often associated with multiple subject designs, first introduced a single subject experimental paradigm in $1945 .{ }^{1}$ Since the introduction, the single subject design has been commonly used within the social and educational sciences. ${ }^{6}$ This design has recently been used as a means of investigation in medicine involving such areas as drug therapy, ${ }^{9}$ gastroenterology, ${ }^{13-15}$ internal medicine, ${ }^{15}$ paediatrics, ${ }^{16}$ family medicine, ${ }^{17}$ cardiology, ${ }^{18}$ nutrition, ${ }^{19}$ and others. ${ }^{16}{ }^{20-22}$ During the 1980s, McMaster University established a service to direct and collaborate with physicians in planning and conducting n-of- 1 trials..$^{20}$ It is reported ${ }^{20}$ that of the 57 completed trials, 50 of those trials provided a definite clinical answer and in 15 cases the study results of the trial resulted in the physician changing the treatment of the patient.

A recent literature example by Langer et al ${ }^{16}$ reports on a single subject randomised trial to assess the effect of cisapride on symptoms arising from gastro-oesophageal reflux in paediatric patients. A placebo and cisapride phase were studied, with three study periods (A-B-A-B-A-B). The outcome variables of interest were number of episodes per five days for vomiting, gagging, and stools. In addition, Guyatt $e a^{22}$ report on a single subject study of a randomised controlled investigation of theophylline. Two study periods of drug and placebo phases were used (A-B-A-B). The symptoms of shortness of breath, the need for an inhaler, and sleep disturbance as patient reported on a seven point scale were used as the outcomes.

At times, single subject research designs have advantages over more traditional group based designs. Some advantages outlined by Franklin $e^{t} a^{25}$ that are especially applicable to practice based family medicine research, include: (1) research situations where research funds are scarce, especially for professionals working in private practice or small clinical settings; (2) research questions that aim to study the process of change; and (3) research questions that are driven by clinical work with the crucial question as to whether a treatment would work for a particular patient. The utilisation of the single subject design for the example above showed these (that is, the data presented in fig 1). This design permitted the investigation of the process of change, both within the baseline and after intervention phases and across these phases, with limited research funds in a private physician office, with the overall intention of answering the research question as to whether this treatment would work for this particular patient.

Limitations of the single subject research design are generalisability of the study conclusions and the methodological and statistical assumptions that are typically needed for inferential statistical tests. ${ }^{43}$ A single subject design provides limited support for conclusions regarding populations of subjects. The results of a single subject design may provide positive findings of the effectiveness of an intervention for a particular subject, however the portion of the 
population that would show this effectiveness and the size of the benefit remain unknown. The non-violation of the methodological and statistical assumptions that are typically needed for inferential tests are difficult to evaluate and test when using a single subject design, because of the limited available data. The evaluation of the validity of the assumptions is more difficult to assess in these small samples. Even with these limitations, estimates and the tests of the effectiveness or intervention effect on the studied subject can be accurately and validly tested using a single subject design.

\section{POSSIBLE RESEARCH QUESTIONS FOR THE PRIMARY CARE PHYSICIAN}

Depending upon the interest of their clinical and patient care needs, the number of research questions that can be addressed by a primary care physician in the practice setting is quite broad. A few examples of possible research questions that can be answered using a single subject research design include:

(1) How does the introduction of a specific antihypertensive drug affect the systolic blood pressure for this patient?

(2) Is there a change in compliance for this patient with twice daily blood glucose readings with the introduction of a reminder system?

(3) What is the optimal dose of a specific antidepressant drug for the control of this patient's self reported symptoms and obtained scores on the Beck depression scale?

(4) Based upon the reported low back and leg pain scores for this patient, does the introduction of an exercise regimen have an impact on those scores?

(5) Does the introduction of an email contact reduce the missed appointments for a particular patient?

(6) Is the level of clinical effectiveness obtained for this particular patient comparable to the results of a recently published clinical trial for the effectiveness of acid suppressive therapy for the control of dyspepsia?

\section{CONCLUSIONS}

With group based research designs, internal and external validity issues need to be considered and balanced. ${ }^{26}$ As the primary questions for a single subject research design concern the investigation of the process of change and whether a treatment would work for a particular patient, internal validity (elimination of bias) issues are paramount. Unfortunately, because of the nature of these designs, external validity (generalisability) when balanced against internal validity is typically left with limited control.

The generalisability of the results from a single subject research study is limited. Possible means of increasing the external validity, generalisability of the results, is by: (1) choosing a subject that is representative of the general type of patients for which this intervention would be used ${ }^{6}$ and (2) by conducting replication studies involving variation in researchers, subjects, or practices. ${ }^{27}$

When planning to implement a single subject design, the specifications necessary to conduct a multiple subject randomised clinical trial must also be followed. The planning phase must incorporate forethought in the choice of the:

(1) outcome variables,

(2) subject,
(3) implementation of the treatment,

(4) number of phases,

(5) number of periods, and

(6) number of observations.

Some guidelines have been prepared for the design of single subject research studies. ${ }^{491624}$ Given the research question of interest, a single subject design should be considered as a means of investigation for practice based primary care research.

Funding: part of this work was supported through a grant from $\mathrm{NIH}$ / NLM R03 LM 8281.

Conflicts of interest: none.

\section{REFERENCES}

1 Edgington ES. Statistics and single case analysis. Prog Behav Modif 1984;16:83-119.

2 McReynolds LV, Thompson CK. Flexibility of single-subject experimental designs. Part 1: review of the basics of single-subject designs. J Speech Hear Disorders 1986;15:194-203.

3 McLaughlin TF. An examination and evaluation of single subject designs used in behavior analysis research in school settings. Educational Research Quarterly 1983;7:35-42.

4 Kazdin AE. Single case research designs: Methods for clinical and applied settings. New York: Oxford University Press, 1982.

5 Cook TD, Campbell DT. Quasi-experimentation: design and analysis issues for field settings. Boston: Houghton Mifflin, 1979.

6 Barlow DH, Hersen M. Single case experimental designs: strategies for studying behavior change. New York: Pergamon, 1984.

7 Kratochwill T, Alden K, Demuth D, et al. further consideration in the application of an analysis-of-variance model for the intrasubject design. J Appl Behav Anal 1974;7:629-34.

8 Larson EB. N-of-1 clinical trials: a technique for improving medical therapeutics. West J Med 1990;152:52-6

9 Guyatt GH, Heyting A, Jaeschke R, et al. N of 1 randomized trials for investigating new drugs. Control Clin Trials 1990;1 1:88-100.

10 Institute of Medicine. Committee on strategies for small-number-participant clinical research trials. Washington, DC: Institute of Medicine, 2001.

11 Kearns KP. Flexibility of single-subject experimental designs. Part II: design selection and arrangement of experimental phases. J Speech Hear Disorders 1986;51:204-14

12 Janosky JE. Use of the nonparametric smoother for examination of data from a single-subject design. Behav Modif 1992; 16:387-99.

13 Lashner BA, Hanquer DB, Silverstein MD. Testing nicotine gum for ulcerative colitis patients: experience with single-patient trials. Dig Dis Sci 1990;35:827-32

14 Woolf GM, Townsend M, Guyatt GH. Treatment of cryptosporidiosis with spiramycin in AIDS: an "N of 1" trial. J Clin Gastroenterol 1987:9:632-4.

15 Balestra DJ, Balestra ST, Wasson JH. Ulcerative colitis and steriod-responsive, diffuse interstitial lung disease: a trial of $\mathrm{N}=1$. JAMA 1988;260:62-4.

16 Langer JC, Wintrop AL, Issenman RM. The single-subject randomized trial: a useful clinical tool for assessing therapeutic efficacy in pediatric practice. Clin Pediatr 1993;32:654-57.

17 Jaeschke R, Cook D, Sackett DL. The potential role of single-patient randomized controlled trials (N-of-1 RCTs) in clinical practice. J Am Board Fam Pract 1992;5:227-9.

18 Robin ED, Burke CM. Single-patient randomized clinical trial: ppiates for intractable dyspnea. Chest 1986;90:888-92.

19 Wagner JL, Winett R. Prompting one low-fat, high-fiber selection in a fastfood restaurant. J Appl Behav Anal 1988;21:179-85.

20 Guyatt GH, Keller JL, Jaeschke R, et al. The n-of- 1 randomized controlled trial: clinical usefulness. Ann Intern Med 1990;112:293-9.

21 Larson EB, Ellsworth AJ, Oas J. Randomized clinical trials in single patients during a 2-year period. JAMA 1993;270:2708-12.

22 Guyatt G, Sackett D, Taylor DQ, et al. Determining optimal therapyrandomized trials in individual patients. N Engl J Med 1986;314:889-92.

23 Janosky JE, Al-Shboul QM, Pellitieri TR. Validation of the use of a nonparametric smoother for the examination of data from a single-subject design. Behav Modif 1995; 19:307-24.

24 Janosky JE, Pellitieri TR, Al-Shboul QM. The need for a revised lower limit for the $4253 \mathrm{H}$, twice nonparametric smoother. Statistics and Probability Letters 1997:32:269-72.

25 Franklin RD, Allison DB, Gorman BS. Design and analysis of single-case research. Mahwah, NJ: Lawrence Erlbaum, 1996.

26 Campbell DT, Stanley JC. Experimental and quasi-experimental designs for research. Boston, MA: Rand McNally College, 1963.

27 Borg WR, Gall MD. Educational research. New York: Longman, 1983. 